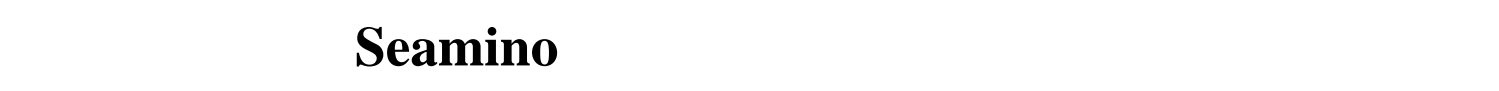 الكيمياوي لحبوب صفين الحن الحظلة
}

\author{
محمد عوبد ألمبيدي \\ عبد العزيزشيخو عبد الجبار عسين صلبر الرلشهي \\ مديرية تربية نينوى \\ قمم علوم الحية \\ الموصل \\ كلية التربية \\ جلمeة الموطل
}

(تاريخ الإتلام 13/ 6 /2011 ؛ تاريخ القبول 26 / / 2011)

\section{الملخص}

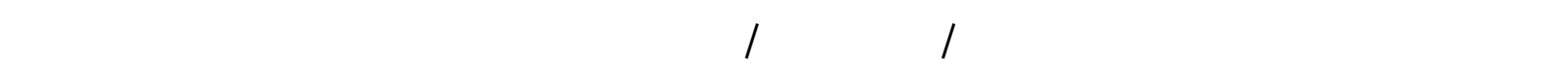

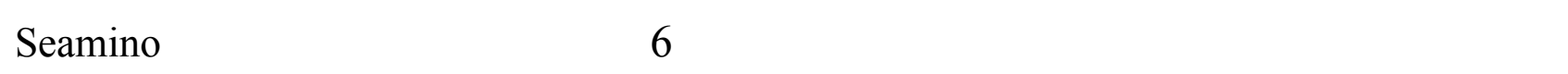

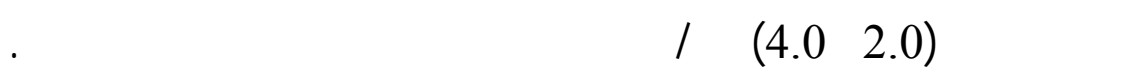

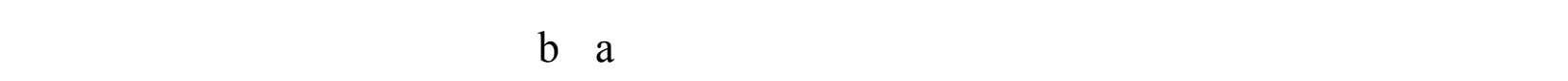

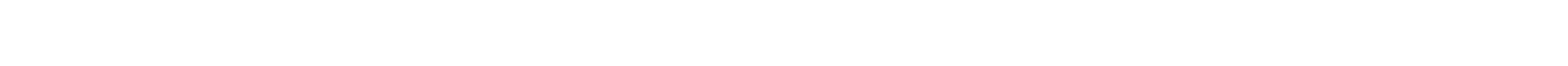
(2.0 و4.0) مل/لتر في حين حصلت زياة معنوية بارتفاع النبلت والمسلحة الورقية والمحتوى الرطوبي في المباتيات

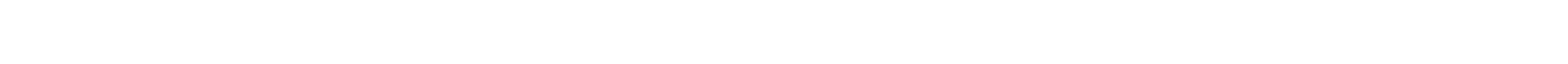
(2.0) aل/لترمن مستخلص العثب مقارنة بمعلملة اللسطرة. كما أوضحت النتائج ققوق الصفشالمك على الصف لم ربيع في اغلب الصفلت المنكورة أعلاه.

الهامل الدالة: العثب البحري، Seamino ، الحظة. 


$$
\text { عبد العزيزشيخو عبد الجبار ولخرون }
$$

\title{
Effect of the Different Seaweed Extract (Seamino) Concentrations on Growth and Seed Chemical Composition of Two Wheat Varieties
}

\author{
Abdul-Aziz S. Abdul-Jabar Hussein S. AL-Rashedy \\ Department of Biology \\ College of Education \\ University of Mosul
}

\author{
Mohammad A. AL-Ubeidi \\ Ninava Directorate of \\ Education \\ Mosul
}

\begin{abstract}
This study conducted at Biology department/ Education college/ Mosul University aiming to evaluate the effect of spraying the shoot parts of two wheat cultivars (Um Rabee and Sham 6) by two Seaweed extracts (Seamino) concentrations (2.0 and 4.0) $\mathrm{ml} / \mathrm{L}$ at three different stages of growth.

The results of this study showed a significant increase in concentration of chlorophyll a and $\mathrm{b}$ in the leave tissues and the $\mathrm{Ca}, \mathrm{Mg}$ and $\mathrm{K}$ content of the cultivated grains during spraying the shoot with $(2.0$ and 4.0$) \mathrm{ml} / \mathrm{L}$ seamino; More over, there was a significant increase in plant length, the relative water content and flare leaf area, but there was a non significant increase in the carbohydrate and protein of the cultivated grains at $(2.0) \mathrm{ml} / \mathrm{L}$ seaweed extract.

The cultivated Sham 6 dominated the cultivated Um Rabee in most parameters studied.
\end{abstract}

Key word: Seaweed, Seamino, Wheat.

\section{الإقمة}

مستخلصك الأعشلب البحرية عبارة عن مستخلصك نباتية تحمل نفس الخصائص الطبيعية للم -واد

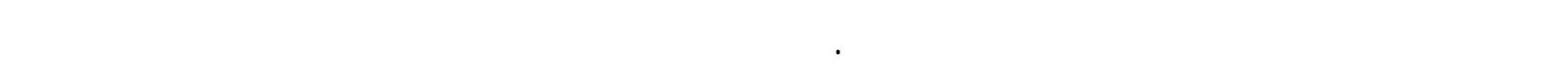

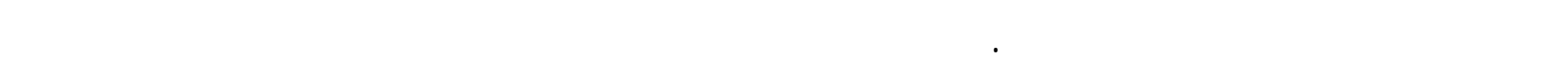

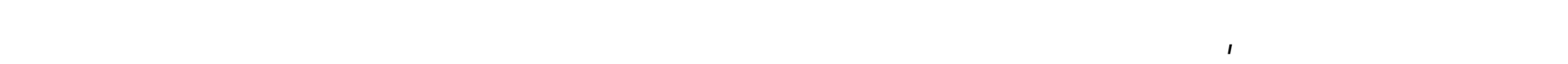

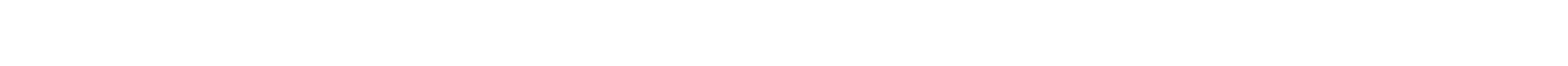

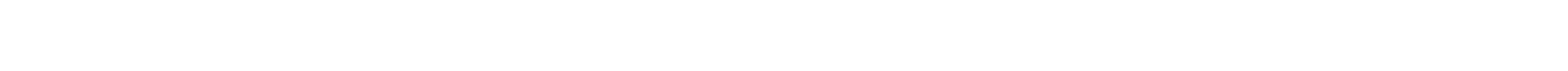

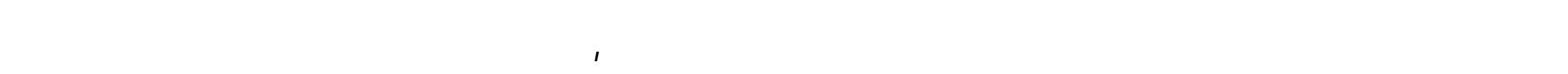

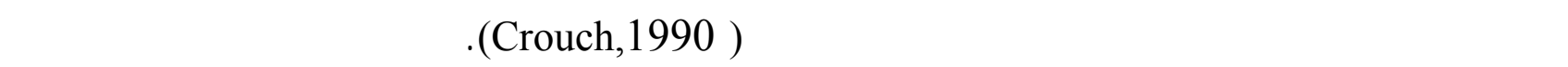

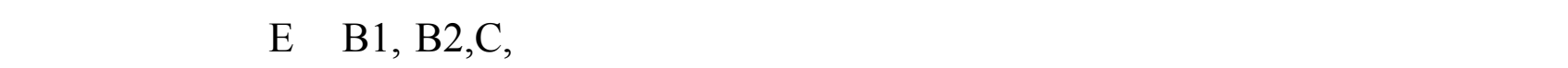
(Moor and Van Staden, 1986) ABA _ _ GAs , cytokinins , Auxins 
(Yan, 1993 ; Nabati et al., وتعمل على تظظم مكونات الخلية وتزيد من تحمل النباتات للجف فاف . 1991)

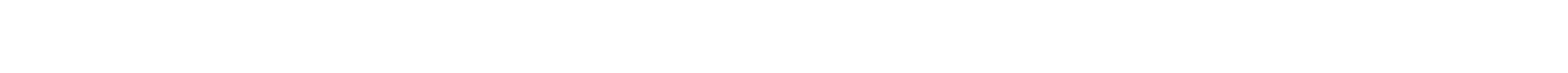
بسب تباين محتواها من العناصر الغذائية الصغرى مل Cu، Zn ، Mo ، Bo ،Co والكبرى ملل Mg وN

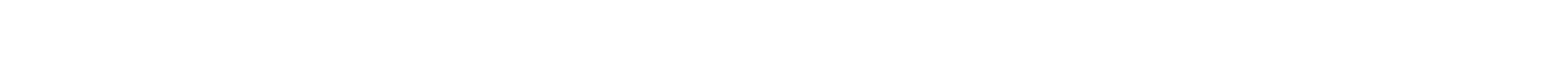

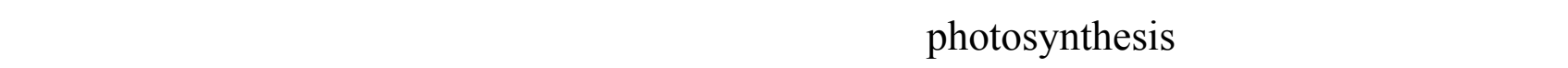
الخضري والجذري وقلة الإصابة بالأمراض وتزيدمن قدرة الجذر على النمو والمتصاص العناصر الغذائي ـة

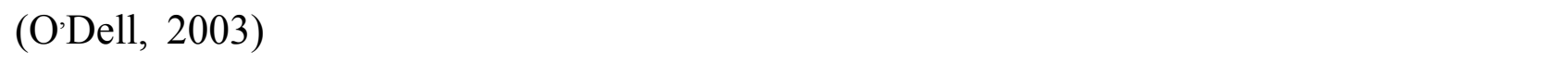
.(Jensen, 2004)

\section{مواد ولرالق المل}

تهيئة الحبوب:

مق الحصول على حبوب صفي الحظة الخشنة لم الربيع وشلم6 المزروعة في العراق م ن ف -رع

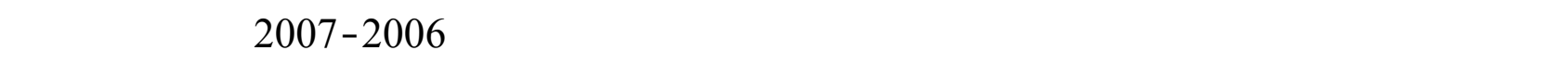
الصفن بالاعتماد على درلسلتساقة وهي من أصنف الحطة الربيعية الغثنة المزروعة ديماً.

تحطلل الترية:

"أخذت التربة على عمق 30سم من مطقة الرشيية في محافظة نينوى، جففت هوائياً وظفت ونعمت

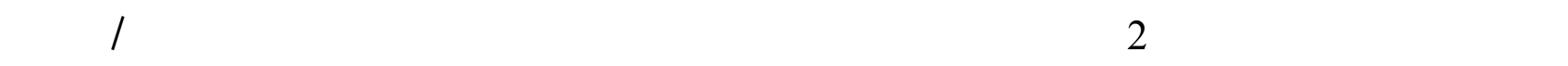
التربية،ومختبرات قم علوم التربة/كلية الزراعة والغابت/جلمعة الموصل إذ قم قيلس نسجه التربة هسب

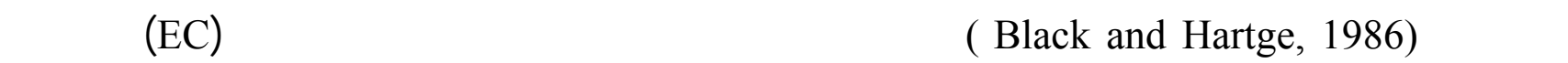

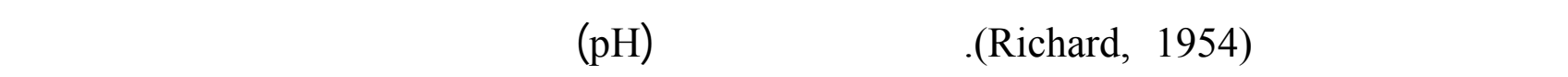

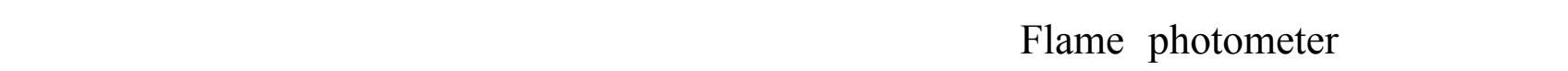
(Black,1965), وكما موضح في الجدول (1). 
عبد العزيزشيخو عبد الجبار ولخرون

الجدول 1: الصفلت الكيميائية والفيزيائية للتربة المستخدة في الدرلسة.

\begin{tabular}{|c|c|c|}
\hline القير & الصفة & ت \\
\hline 32.0 & الرaل (\%) & 1 \\
\hline 38.8 & الغرين (\%) & 2 \\
\hline 29.2 & الطين (\%) & 3 \\
\hline مزيجيةطينية & النسجة & 4 \\
\hline 1.30 & المادة العضوية (\%) & 5 \\
\hline 0.75 & درجة التوصيل الكهربائي (E.C) سيسيسيمنز /م & 6 \\
\hline 7.42 & درجة قفاعل التربة (pH) & 7 \\
\hline & الايونات الذائبة مليمكلفء/ لتر & 8 \\
\hline $\begin{array}{c}0.8 \\
0.63 \\
1.4 \\
0.66 \\
1.7\end{array}$ & 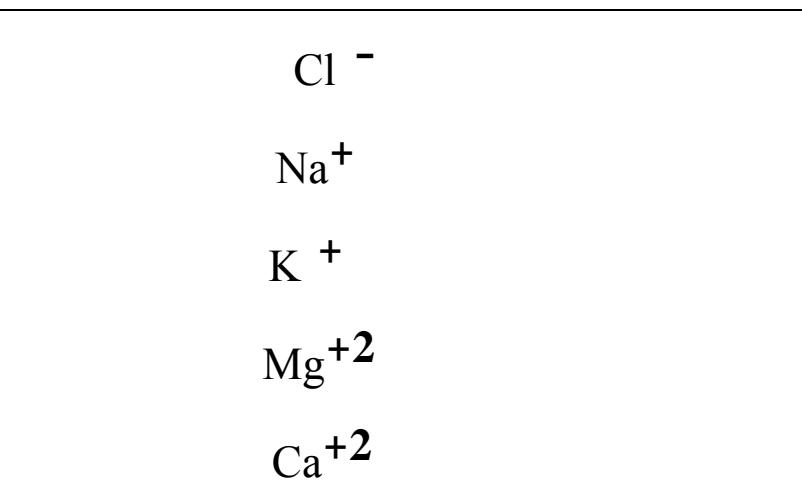 & \\
\hline
\end{tabular}

قدير السعة الهقلية للتربة:

مق تقدير اللسعة الققلية للتربة المستخمة في التجربة بأخخذ علبة صغيرة مقفبة القاعة ومعروف ـة الـ -وزن

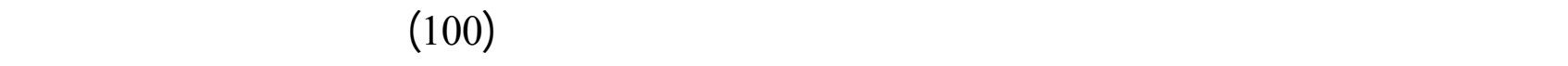

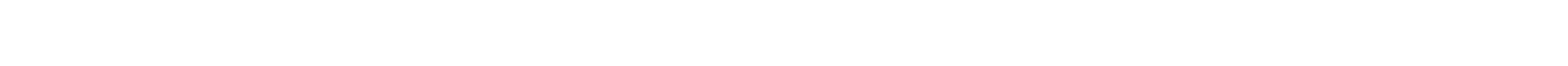

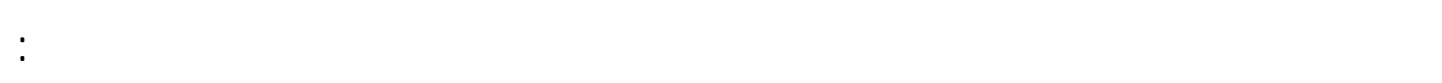

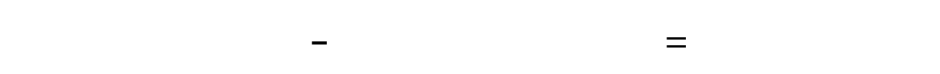
وزن ماء التربة

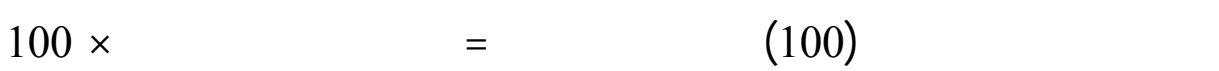
وزن التربة الجلفة

المعاملات المستخمة:

قم وش المجلمبع الخضرية لصفي الحظة الخشنة لم الرببع وشلم6 في تجربة البي ـت البلمه ـتيكي

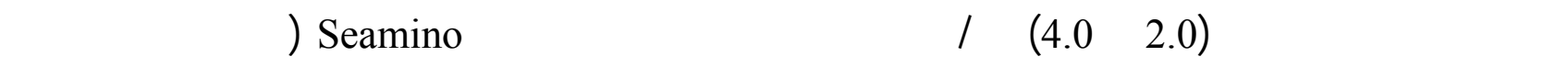




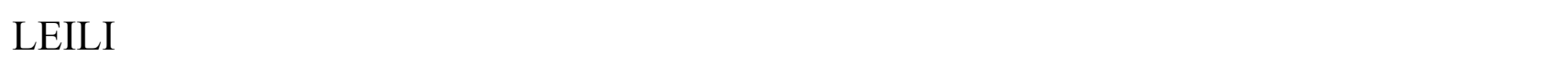

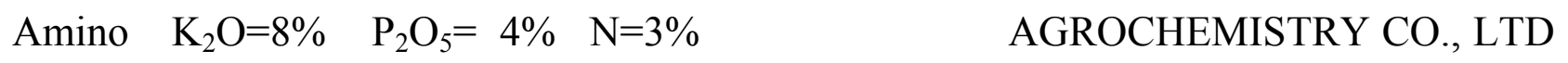
Seaweed Extract =18\% gacid =10\%

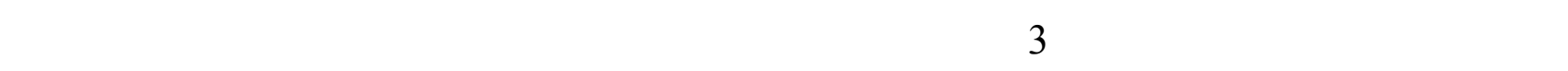

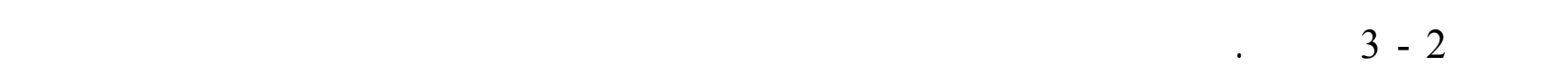

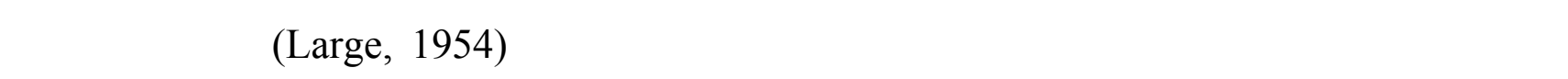

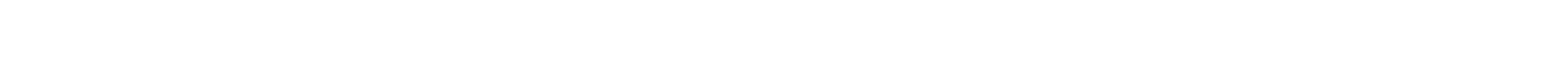

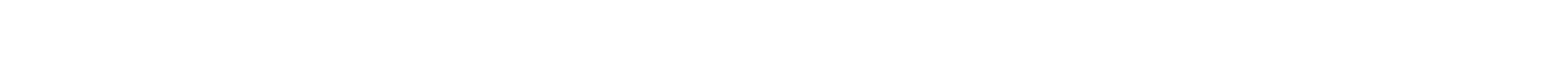

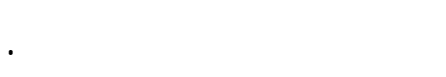

\section{الزراعة والري:}

زرعت بذور الحظة في 2010/12/22 بواقع (15) بذرة/أصيص، وقمراعاعت أن تكون المسفلت

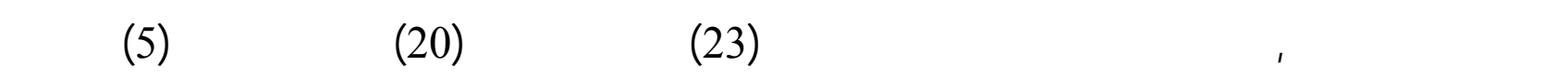

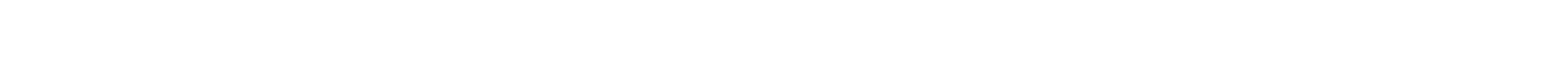

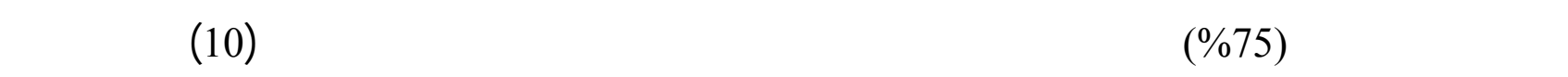
الزراعة خفف عدد البادرات إلى (5) بادرات في كل أصيص, مق قيلس المسلحة الورقية وتركيز الكلوروفل الميل

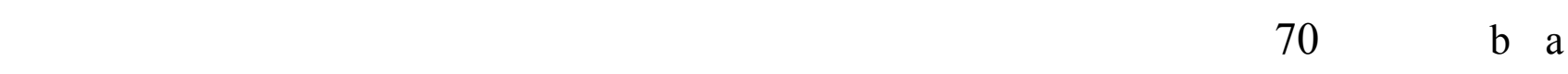

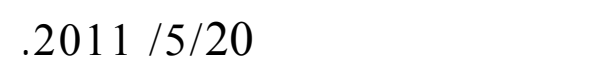

المفلت المدروصة قيلس الرفاع النبلت و المساحة الورقية:

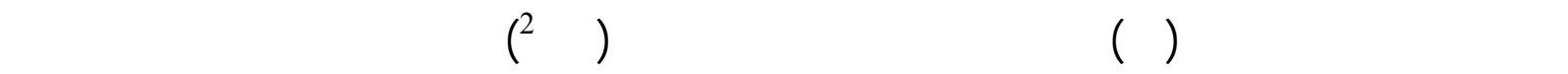

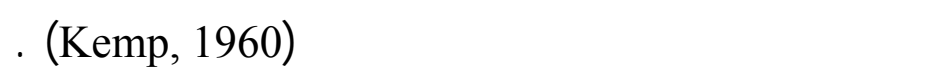

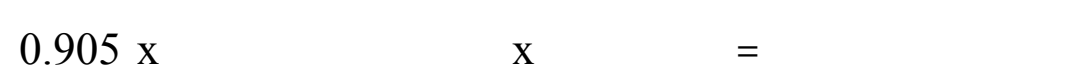
فيرمحتوى الماء النسبي في الأنسجة الورقية الورة

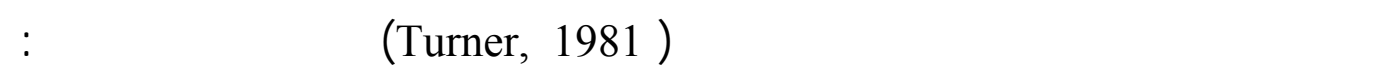
الوزن الطري - الوزن الجف $100 \times$ محتوى الماء النسب (\%) =

الوزن ألامتلائي - الوزن الجل 


\section{فديرمحتوى الكلوروفلف الأورق:}

قدر الكلوروفيل في الأوراق بعهبطوريقة (Arnon, 1949 ; Makinny, 1941) إذ م لخذ الأوراق النباتيي ـة

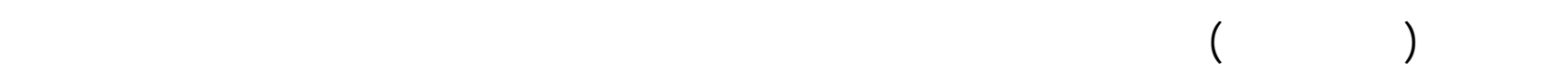

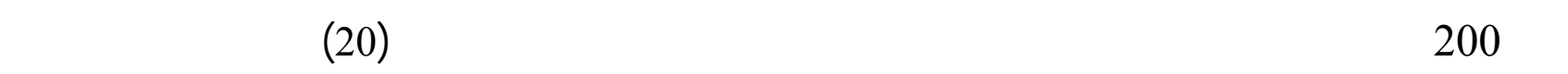
بتركيز(80\%) وفصل الرلشح كن الرلسب المتقي بوسطة جهاز الطرد المركزي من نوع ( Hettich EBA)

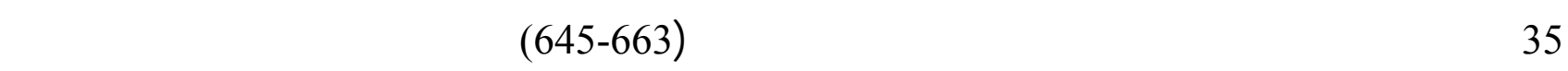

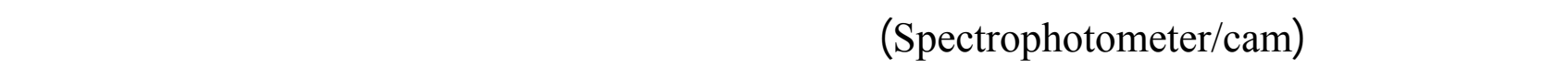
(A , B ) (A

Chl.a $=(12.7($ D 663) $-2.69($ D 645) $) \times \mathrm{V} /(1000 \times \mathrm{W})$.

$\mathrm{Chl} . \mathrm{b}=(22.9(\mathrm{D} 645)-4.68(\mathrm{D} 663)) \times \mathrm{V} /(1000 \times \mathrm{W})$.

تر قراءة الكثلفة الضوئية للكلوروفيل المستخلص على الأطول الموجية 663 و645 نانوميتر على التوالي.

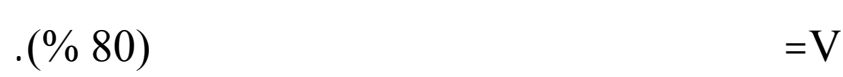
=W الوزن الرطب بالغرالم للنسبج النباتي الذي مة لستخلاصه.

\section{قدير الكاريوهيدرات:}

قدرت كمية الكاربوهيدرات في حبوب نباتنات الحطة المزروعة في البيت البلمستيكي، تبعا لطريق ـة (Herbert et al., 1971) فوفلت الكاربوهيدرات المذلبة في الرلشح عن الرلمب بلستخدلم جهاز الطرد المركزي من نوع ( Hettich (EBA35 وق ققدير الكاربوهيدرات بلستعمل طريقة الفينول -حلمض الكبريتيك بولنطة قيلس الكثلفة المرئية عند الطول ألموجي (488) نانوميتر بلستعمل جهاز الطير بلسيف الضورئي.

\section{قحير البروتين:}

قم ققدير البروتين في حبوب نباتلت الحطة بأتباعطريقة ف -ولن (Schacterale and Pollak, 1973)

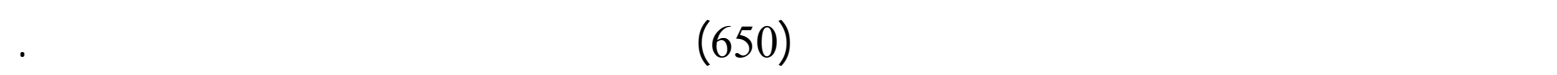
قدير العناصر الغذائية:

قم تقدير العناصر الغذائية في حبوب نباتلت الحطة، إذ لخذ العينات النباتية المجفة ـة مـ ن الحب -وب

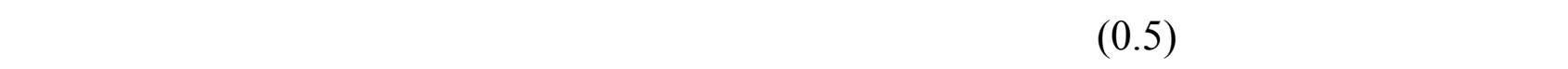
وم ققدير البوتلسيوم Kapman and Partt, 1961) 
والكللسيوم Ca (Flam Photometer) .(Richard, 1954 )

\section{النحلل الإحصائي:}

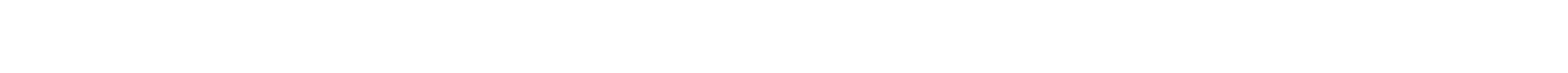
Completely Randomized Design (C.R.D)

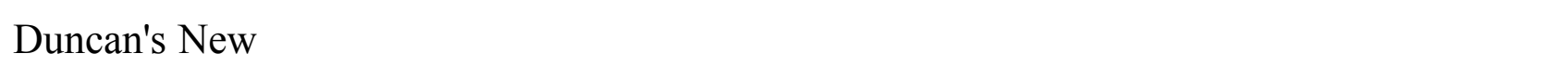
.) ( Duncan, 1955) Multiple Range Test

\section{النتئيج والمنالثشة}

يتضح من نتائج الجدول (2) حصول زيادة غير معنوية بطول المجموع الخضري وزيادة معنوي ة في المسلحة الورقية والمحتوى الرطوبي للأنسجة الورقية في نباتتا الحطة عند لمنتخدلم التركيز 4 مل/لتر

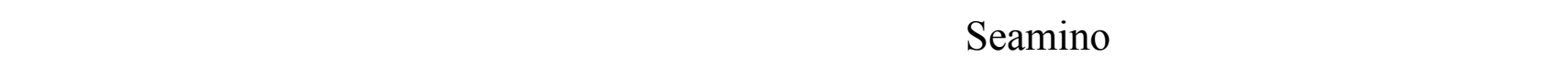

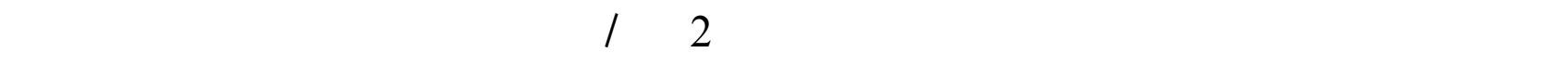

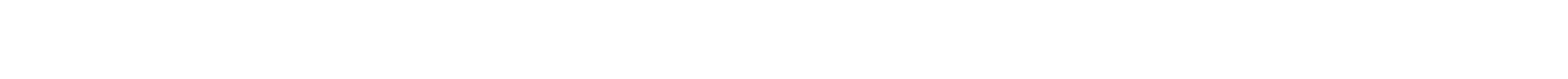

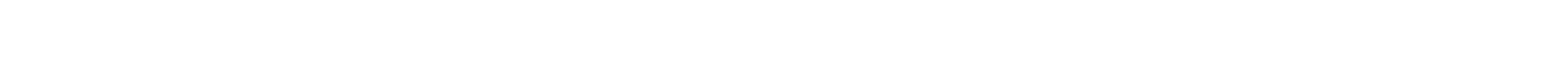

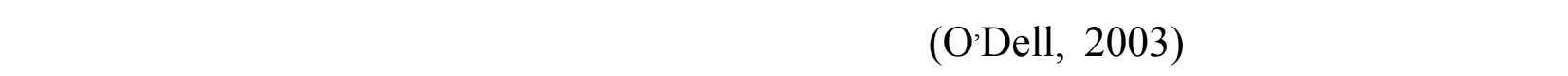

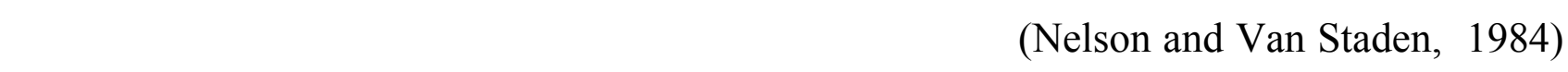

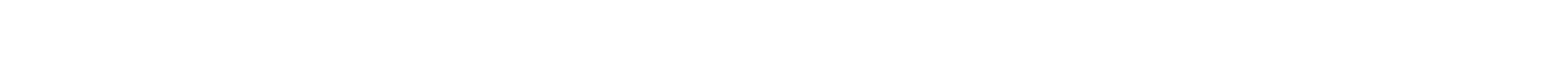

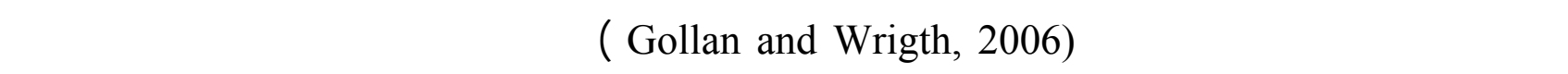

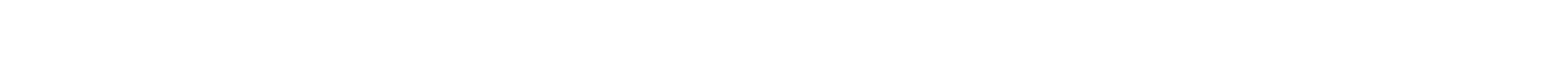

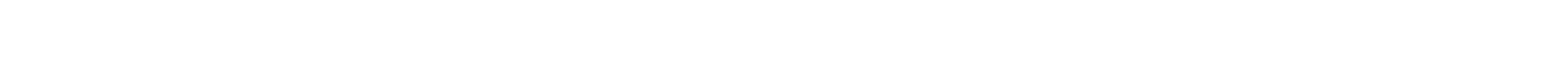

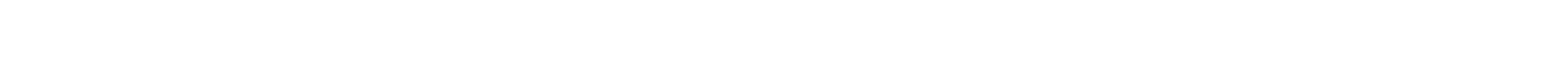

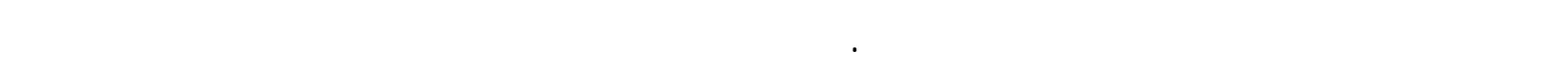

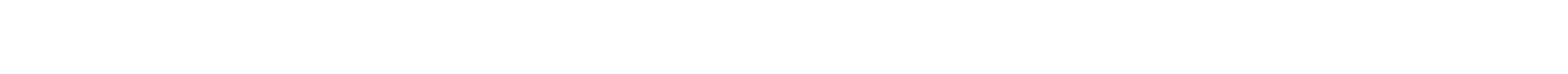

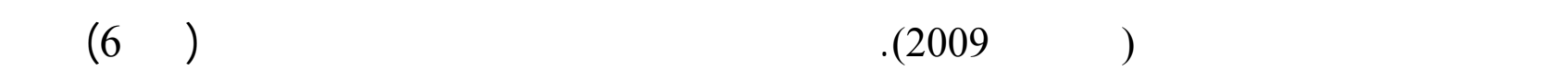

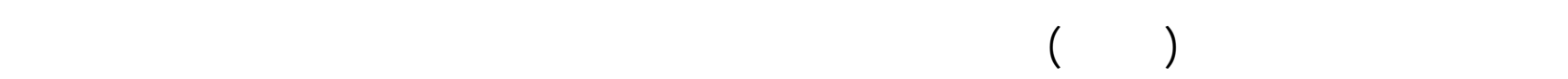
معنوية بين الصفين في صفة المحتوى الرطوبي للأنسجة الورقية. 


$$
\text { عبد العزيزشيخو عبد الجبار ولخرون }
$$

الجدول 2: تأثير لستخدلم ترلكيز مختلفة من مستخلص العثب البحري Seamino على ارقفاع النبت والمسلحة الورقية والمحتوى الرطوبي للأنسجة الورقية لصفين من نباتلت الحظة.

\begin{tabular}{|c|c|c|c|c|c|c|c|c|c|c|}
\hline المعالملات & تألثير & المحتوي \% & ألثير & تألثير & الورقةلسم2 & المعالملات & الأصناف & النبلتطبم & المعاملات & الأصنلف \\
\hline 48.739 b & \multirow{3}{*}{55.259 a } & 53.000 bc & $23.023 \mathrm{~b}$ & \multirow{3}{*}{$20.467 \mathrm{~b}$} & 23.355 b & $67.650 \mathrm{ab}$ & \multirow{3}{*}{$65.669 \mathrm{~b}$} & $65.647 \mathrm{ab}$ & اللسيلرة & \multirow{3}{*}{ لم ربع } \\
\hline $52.618 \mathrm{~b}$ & & 56.297 b & $26.888 \mathrm{ab}$ & & 18.127 b & $65.150 \mathrm{~b}$ & & $63.900 \mathrm{~b}$ & $\begin{array}{c}\text { Seamino } \\
2 \mathrm{ml} / \mathrm{L}\end{array}$ & \\
\hline 63.349 a & & 56.480 b & 28.775 a & & $19.919 \mathrm{~b}$ & 68.710 a & & $67.460 \mathrm{ab}$ & $\begin{array}{c}\text { Seamino } \\
4 \mathrm{ml} / \mathrm{L}\end{array}$ & \\
\hline & \multirow{3}{*}{54.545 a } & $44.478 \mathrm{~d}$ & & \multirow{3}{*}{$31.990 \mathrm{a}$} & 22.691 b & & \multirow{3}{*}{68.671 a } & 69.653 a & اللسلرة & \multirow{3}{*}{ شالم 6} \\
\hline & & $48.939 \mathrm{~cd}$ & & & 35.648 a & & & $66.400 \mathrm{ab}$ & $\begin{array}{c}\text { Seamino } \\
2 \mathrm{ml} / \mathrm{L}\end{array}$ & \\
\hline & & 70.217 a & & & $37.630 \mathrm{a}$ & & & $69.960 \mathrm{a}$ & $\begin{array}{c}\text { Seamino } \\
4 \mathrm{ml} / \mathrm{L}\end{array}$ & \\
\hline
\end{tabular}

المعدلات ذات الأحرف المشابهة لا تختلف معنويا عند مستوى الاحتمل (5\%) بحسب لختبار دنكن متعدد الحدود. 


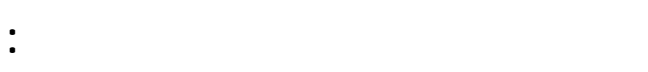

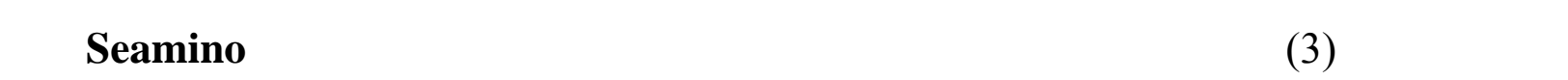

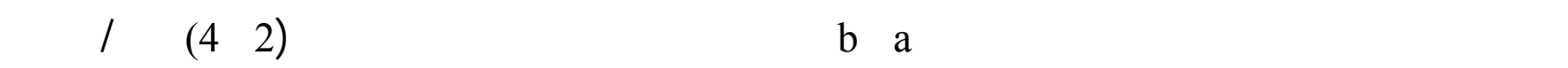

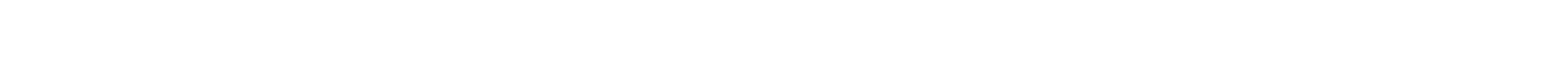

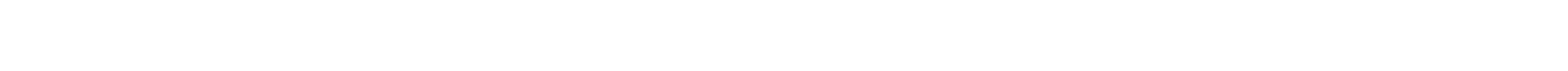
النيتروجين والمغنيسيوم فضلاً عن الحيد الذي يساعد في بناء الكلوروفيل. ( الصحلف, 1989).

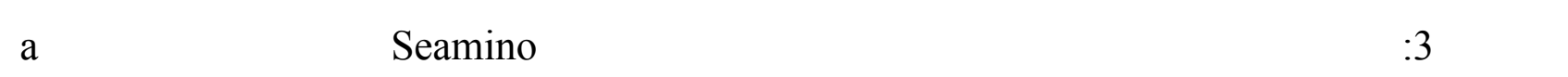

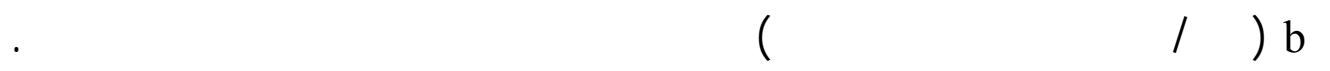

\begin{tabular}{|c|c|c|c|c|c|c|c|}
\hline المعالمالات & الأصنلف تأثير & كلورولفل & المعاملات & لأصنألفير & كلوروفل & المعاللات & الأصنف \\
\hline $0.524 \mathrm{c}$ & \multirow{3}{*}{$0.618 \mathrm{~b}$} & $0.555 \mathrm{c}$ & $1.435 \mathrm{~b}$ & \multirow{3}{*}{$1.889 \mathrm{a}$} & $1.363 \mathrm{~b}$ & للسطرة & \multirow{3}{*}{ لم ربيع } \\
\hline $0.718 b$ & & $0.555 \mathrm{c}$ & $2.076 \mathrm{a}$ & & $2.110 \mathrm{a}$ & $\begin{array}{l}\text { Seamino } \\
2 \mathrm{ml} / \mathrm{L}\end{array}$ & \\
\hline \multirow[t]{4}{*}{$0.833 \mathrm{a}$} & & $0.743 \mathrm{~b}$ & $2.109 \mathrm{a}$ & & $2.183 \mathrm{a}$ & $\begin{array}{l}\text { Seamino } \\
4 \mathrm{ml} / \mathrm{L}\end{array}$ & \\
\hline & \multirow{3}{*}{$0.766 \mathrm{a}$} & $0.493 \mathrm{c}$ & & \multirow{3}{*}{$1.859 \mathrm{a}$} & $1.506 \mathrm{~b}$ & للسطرة & \multirow{3}{*}{ شلم 6} \\
\hline & & $0.882 \mathrm{a}$ & & & $2.035 \mathrm{a}$ & $\begin{array}{c}\text { Seamino } \\
2 \mathrm{ml} / \mathrm{L}\end{array}$ & \\
\hline & & $0.922 \mathrm{a}$ & & & $2.035 \mathrm{a}$ & $\begin{array}{c}\text { Seamino } \\
4 \mathrm{ml} / \mathrm{L}\end{array}$ & \\
\hline
\end{tabular}

المعدلات ذات الأحرف المتشبهة لا تختلف معنويا عندمستوى الاحتمل (5\%) بهنب لختبار نكن متعدد الحدود.

كما تبين من نتائج الجدول (4) إن لستخده مستخلص العشب البحري أدى إلى حصول زي ادة غي ـر

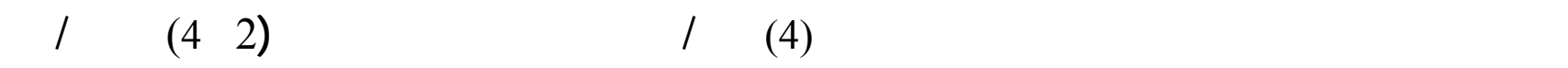

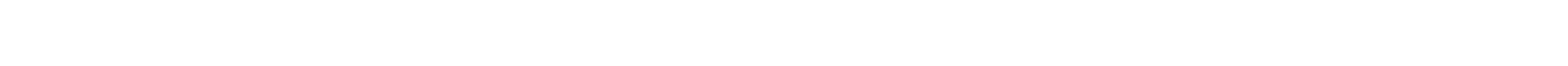

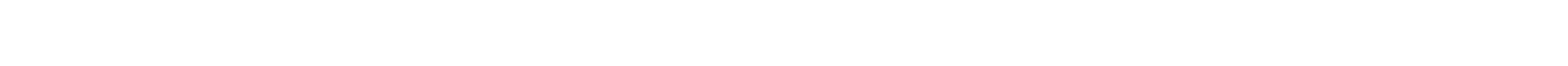

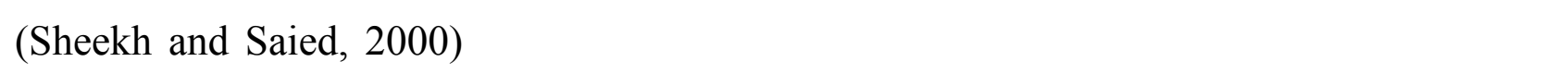

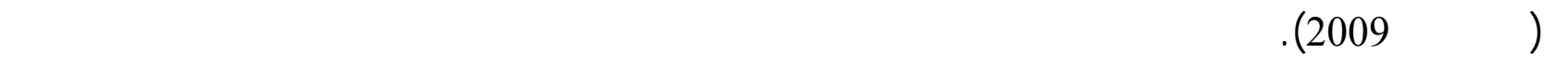

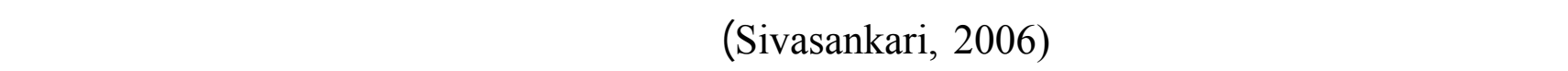




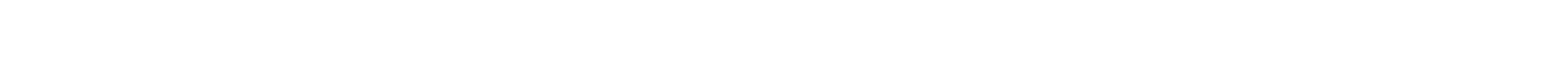
المستخلصت تحوي على ترلكيز عالية منه والتي لها دوركبير في بناء البروتينت (Staden et al., 1994)

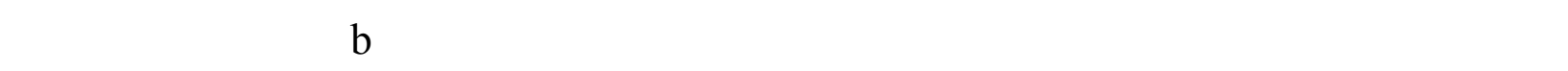
الصف (شلم 6) وعلى العكس من ذلك فقد لوظ حصول قفوق معنوي للصف (لم ربيع) بتركيز البروتين. الجدول 4: تأثير لستخدلم ترلكيز مختلفة من مستخلص العثب البحري Seamino على تركيز الكاربوهيدرات والبروتينت (ملغم /غم ) في حبوب صفين من نباتلات الحطة.

\begin{tabular}{|c|c|c|c|c|c|c|c|}
\hline $\begin{array}{c}\text { المعالثير } \\
\text { المعلات }\end{array}$ & $\begin{array}{l}\text { الأصناف } \\
\text { تأثير }\end{array}$ & البروتينات & |المعاملات & الأصنأفير & الكارونهيرات & المعاملات & الأصناف \\
\hline $4.108 \mathrm{a}$ & \multirow{3}{*}{$4.675 \mathrm{a}$} & $4.640 \mathrm{a}$ & $\begin{array}{c}55.779 \\
a\end{array}$ & \multirow{3}{*}{$\begin{array}{c}48.554 \\
\mathrm{~b}\end{array}$} & $47.785 \mathrm{c}$ & للسططرة & \multirow{3}{*}{ لم ربيع } \\
\hline $4.211 \mathrm{a}$ & & $4.681 \mathrm{a}$ & $\begin{array}{c}53.580 \\
\mathrm{a}\end{array}$ & & $48.632 \mathrm{c}$ & $\begin{array}{r}\text { Seamino } \\
2 \mathrm{ml} / \mathrm{L}\end{array}$ & \\
\hline \multirow[t]{4}{*}{$4.234 \mathrm{a}$} & & $4.705 \mathrm{a}$ & $\begin{array}{c}58.364 \\
\mathrm{a}\end{array}$ & & $49.244 \mathrm{c}$ & $\begin{array}{r}\text { Seamino } \\
4 \mathrm{ml} / \mathrm{L}\end{array}$ & \\
\hline & \multirow{3}{*}{$3.693 \mathrm{~b}$} & $3.575 \mathrm{~b}$ & & \multirow{3}{*}{$\begin{array}{c}63.262 \\
\mathrm{a}\end{array}$} & $63.773 \mathrm{ab}$ & اللسطرة & \multirow{3}{*}{ شلم 6} \\
\hline & & $3.740 \mathrm{~b}$ & & & $58.528 \mathrm{~b}$ & $\begin{array}{r}\text { Seamino } \\
2 \mathrm{ml} / \mathrm{L}\end{array}$ & \\
\hline & & $3.764 \mathrm{~b}$ & & & $67.484 \mathrm{a}$ & $\begin{array}{r}\text { Seamino } \\
4 \mathrm{ml} / \mathrm{L}\end{array}$ & \\
\hline
\end{tabular}

المعدلات ذات الأحرف المتشابهة لا تختلف معنويا عندمستوى الاحتمل (5\%) بهمب لختبار نكن متعدد الحدود.

\section{تركيز بصض الهناهر الغذائية في الحبوب :}

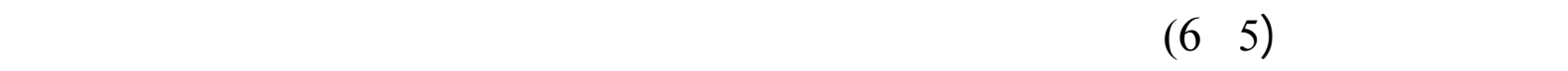
لستخدلم مستخلص العثب البحري عند التركيزين (2 و4 ) مل /لتر مقارنة بمعلملة للسيطرة، وهذه النت ائج

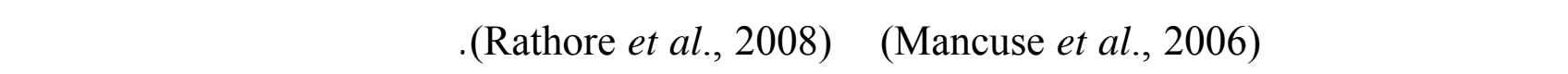

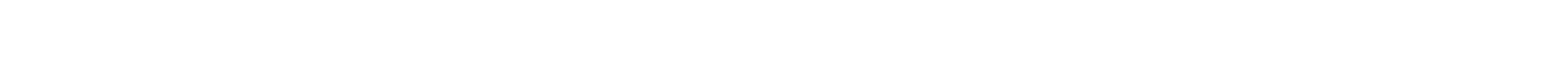

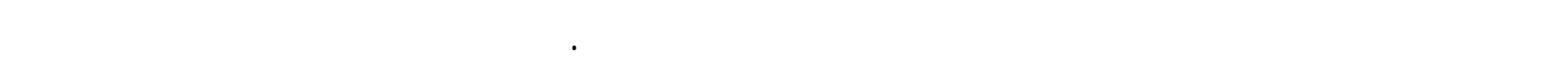

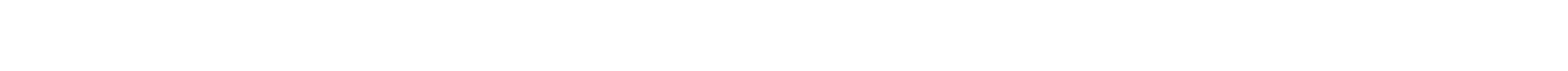

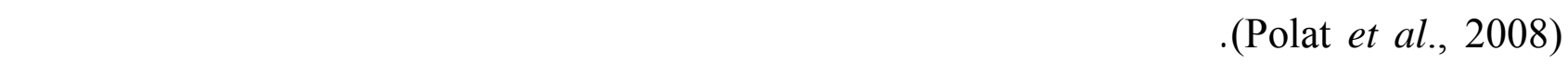
زيادة لمتصاص العناصر المغنية وزيادة تركيزها في النبل (Jensen , 2004) و(العبيدي, 2009). 
في حين لوظظ حصول النخاض معنوي بتركيز الصوديوم في الحبوب عند لستخدلم مستخلص العثب

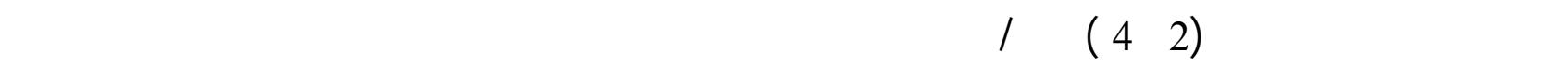

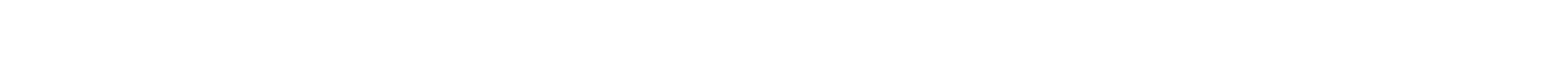
الخلايا (ألنعيمي, 2000 ). كذلك لوهظ عدم وجود لختلافلت معنوية بين الصفين في تركيز الكللسيوم والمغني سيوم والبوتلد يويو ف مي

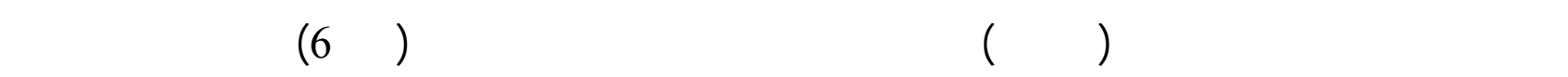

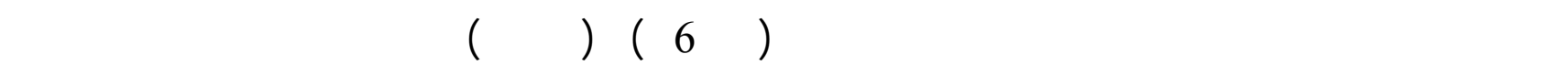

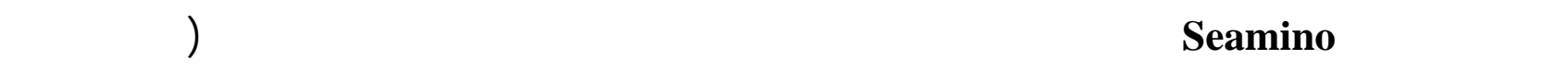

الجدول 5: تأثيرلمتخدلم ترلكيزمختلفة من مستخلص العثب البحري Seamino عل مق تركي -ز الكال سيوم والمغنيسيوم (\%) في حبوب صفن من نباتتات الحطة.

\begin{tabular}{|c|c|c|c|c|c|c|c|}
\hline المعاملات & لأصناd & المغنيسيقو & المعالملات & لأصناف & |كالسيوم & الممالات & لأصناف \\
\hline $2.183 \mathrm{~b}$ & \multirow{3}{*}{$2.496 \mathrm{a}$} & $2.087 \mathrm{~b}$ & $2.375 \mathrm{~b}$ & \multirow{3}{*}{$2.583 \mathrm{a}$} & $\begin{array}{c}2.450 \\
\text { bc }\end{array}$ & للسططرة & \multirow{3}{*}{ لم ربيع } \\
\hline $2.705 \mathrm{a}$ & & $2.460 \mathrm{~b}$ & $2.800 \mathrm{a}$ & & $\begin{array}{c}2.600 \\
b c\end{array}$ & $\begin{array}{r}\text { Seamino } \\
2 \mathrm{ml} / \mathrm{L}\end{array}$ & \\
\hline \multirow[t]{4}{*}{$2.940 \mathrm{a}$} & & $2.940 \mathrm{a}$ & $2.967 \mathrm{a}$ & & $\begin{array}{c}2.700 \\
\mathrm{abc}\end{array}$ & $\begin{array}{r}\text { Seamino } \\
4 \mathrm{ml} / \mathrm{L}\end{array}$ & \\
\hline & \multirow{3}{*}{$2.723 \mathrm{a}$} & $2.280 \mathrm{~b}$ & & \multirow{3}{*}{$2.844 \mathrm{a}$} & $2.300 \mathrm{c}$ & للسططرة & \multirow{3}{*}{ شd 6} \\
\hline & & $2.950 \mathrm{a}$ & & & $\begin{array}{c}3.000 \\
\mathrm{ab}\end{array}$ & $\begin{array}{c}\text { Seamino } \\
2 \mathrm{ml} / \mathrm{L}\end{array}$ & \\
\hline & & $2.940 \mathrm{a}$ & & & $3.233 \mathrm{a}$ & $\begin{array}{r}\text { Seamino } \\
4 \mathrm{ml} / \mathrm{L}\end{array}$ & \\
\hline
\end{tabular}

المعدلات ذات الأحرف المتشبهة لا تختلف معنويا عند مستوى الاحتمل (5\%) بهسب لختبار نكن متعدد الحدود. 
الجدول 6: تأثير لمتخدلم ترلكيزمختلفة من مستخلص العثب البحري Seamino على تركي ـز ال صوديوم

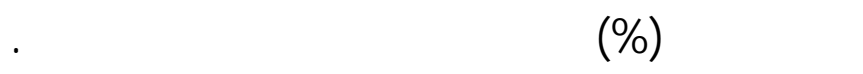

\begin{tabular}{|c|c|c|c|c|c|c|c|}
\hline المعاملات & الأصناف & البوتلسيوم & المعاملات & الأصناف & الصويو & المعالالت & الأصنا \\
\hline $3.219 \mathrm{c}$ & \multirow{3}{*}{$3.550 \mathrm{a}$} & $3.166 \mathrm{c}$ & $1.192 \mathrm{a}$ & \multirow{3}{*}{$1.253 \mathrm{a}$} & $1.359 \mathrm{a}$ & للسطرة & \multirow{3}{*}{ لم ربيع } \\
\hline $3.579 \mathrm{~b}$ & & $3.559 \mathrm{~b}$ & $0.872 \mathrm{~b}$ & & $1.237 \mathrm{~b}$ & $\begin{array}{r}\text { Seamino } \\
2 \mathrm{ml} / \mathrm{L}\end{array}$ & \\
\hline \multirow[t]{4}{*}{$3.958 \mathrm{a}$} & & $3.927 \mathrm{a}$ & $0.842 \mathrm{~b}$ & & $1.176 \mathrm{~b}$ & $\begin{array}{r}\text { Seamino } \\
4 \mathrm{ml} / \mathrm{L} \\
\end{array}$ & \\
\hline & \multirow{3}{*}{$3.620 \mathrm{a}$} & $3.273 \mathrm{c}$ & & \multirow{3}{*}{$0.679 \mathrm{~b}$} & $1.024 \mathrm{c}$ & للسطرة & \multirow{3}{*}{ شلم 6} \\
\hline & & $3.600 \mathrm{~b}$ & & & $0.507 \mathrm{~d}$ & $\begin{array}{r}\text { Seamino } \\
2 \mathrm{ml} / \mathrm{L}\end{array}$ & \\
\hline & & $3.988 \mathrm{a}$ & & & $0.508 \mathrm{~d}$ & $\begin{array}{r}\text { Seamino } \\
4 \mathrm{ml} / \mathrm{L}\end{array}$ & \\
\hline
\end{tabular}

المعدلات ذات الأحرف المتشبهة لا تختلف معنويا عندمستوى الاحتمل (5\%) بهسب لختبار نذكن متعدد الحدود.

\section{المصادر المربية}

التميمي، جميل يلسين علي (2009) . تأثير حلمض الهيوميك ومستخلصك الطحالب البحري ـة ف مي النم -و

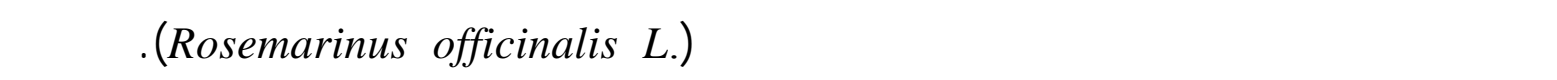
مقبول للنثر في مجلة تكريت للعلوم الصرفة. للساهوكي،مدت وهيب؛محمد, كريمة (1990). طبيقلت في تصميم وتحليل التج ارب، مطبع لة دار الحكمة الطباعة والنثر، الموصل. مجدم، الصحل، فاطل مسين (1989). أظمة الزراعة بدون لستخدلم تربة. مطبعة دار الحكم ة. جلمع ـة بغ ـداد, وزارة التعليم العالي والبهث العلمي ــأجمهورية العراق.

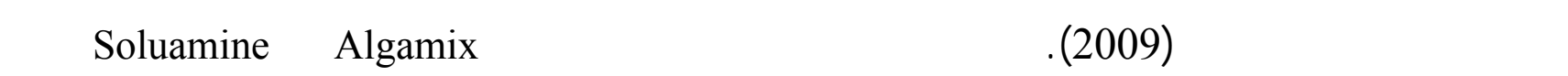

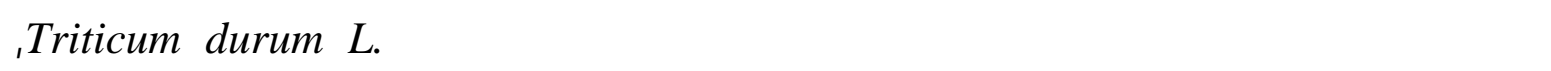
بسالة ملجستير, كلية التربية, جلمعة الموصل.

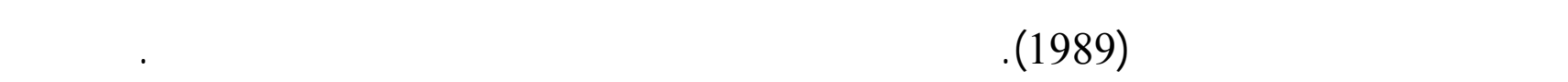
ملجستير, كلية العلوم, جلمعة الموصل. 


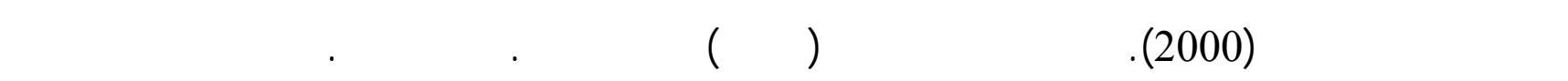
الكتب الطباعة والنشر، جلمعة الموطل.

\section{المصادر الأجنبية}

Arnon, D. I. (1949). Copper enzyme in isolated chloroplastes polyphenol oxidase in Beta vulgaris., Plant Physiol., 24, 1-15.

Black, C. A . (1965). "Methods of Soil Analysis" . Part 2. Amer. Soc. Agron . Inc . U.S.A.

Black, G. R.; Hartge, C. (1986). Bulk density in methods of soil structure and migration of colloidal materials in soil. Soil Sci . Soc . Am. Proc ., 26, 297-300.

Chapman, H.D.; Partt, P. F. (1961). Methods of analysis for soil, plant and water. Univ of Calif . Div. Agric. Sci.

Crouch, I. J. (1990). The effect of seaweed concentrate on plant growth. Dissertation for doctor of philosophy. Dept. Botany. Univ. Natal, Pietermaritzburg. South Africa.

Duncan, D.P. (1955). Multiple range and multiple F-Test . Biometrics, 11,1-42.

Gollan, J. R. ; Wright, J. T. (2006). Limited grazing pressure by native herbivores on the invasive seaweed Caulerpa. taxi folia in atemprate. Australia estuary marine and Freshwater Research., 57(7), 685-694.

Herbert, D.; Philips, P. J.; Strange, R.E. (1971). "In Methods in Microbiology". Norries, J.R. and Robbins, D.W.(eds.) Acad. , press, London and New York. 5B. Chap.3.

Jensen, E. (2004). Seaweed; Fact or Fancy. From the Organic Broadcaster, Published by moses the Midwest Organic and Sustainable Eductation. From the Broadcaster, 12(3), 164-170.

Kemp, C. D. (1960). Method of estimating the leaf area of grasses from liner measurements. Ann. Bot., Lon. 24(96), 491- 499.

Large, E. C. (1954). Growth stages in cereals illustration of the feekes scale. Plant Pathology., 3, 127 - 129.

Makinny, G. (1941). Absorption of light by chlorophyll solution. J. Biol. Chem.,140, 315322.

Mancuso, S. ; Azzarello, E. ; Mugnai, S. ; Briand, X. ( 2006). Marine bioactive substances (IPA extract) improve foliar ion uptake and water tolerance in potted Vitis vinifera plants. Advances in Horticultural Science, 20, 156-161.

Moore, P. A.; Van Staden, J . ( 1986 ). Algae and cytokinins. J. Plant Physiology, 123,1-2.

Nabati, D. A.; Schmidt, R. E.; Parrish, D.J. (1991). Effect of PGR product and Fe on waterstressed Kentucky bluegrass. Proc. Plant Growth Regulator Soc. Amer., 170171.

Nelson, W. R.; Van Staden, J. (1984). The effect of seaweed concentrate on wheat Culms. J. Plant Physiology, 115, 433-437.

O'Dell, C. (2003). Natural plant hormones are biostimulants helping plants develop plant antioxidant activity for multiple benefits. Virginia Vegetable, Small Fruit and Special Crops., 2(6), $1-3$. 
Polat, E. , Demir, H.; Onus, A. N. ( 2008 ). Comparison of some yield and quality criteria in organically and conventionally grown lettuce. African $J$. Biotechnology. 7 (9), 1235 - 1239.

Rathore, S. S.; Chaudhary, D. R.; Boricha, G. N.; Ghosh, A.; Bhatt, B. P.; Zodape, S. T.; Potolia, J. S. (2008). Effect of seaweed extract on the growth, yield and nutrient uptake of Soybean (Glycine max) under rainfed conditions. South African J. Botany. 382, $5-9$.

Richard, I. A. (1954). Diagnosis and Improvement of Salience and Alkali Soil. U. S. Dept. Agric. Handbook.

Schacterale, G. R. ; Pollak, R.L. (1973). A simplified methods for the quantitative assay of small amount of protein in biological material. Anal. Bio. Chem., 51, 651-655.

Sivasankari, S.; Venkatesaln, V.; Anantharaj, M.; Chandrasekaran, M. (2006). Effect of seaweed extract on the growth and biochemical constituents of Vigna sinensis. Bioresource Technology, 97, $1745-1751$.

Sheekh, M. M. ; Saied, A. D. (2000). Effect of crude seaweed extracts on seed germination, seedling growth and some metabolic processes of Vicia faba $\mathrm{L}$. Cytobios ., (396), $23-35$.

Staden, J. Van; Upfold, S., J. ; Drewes, F. E. (1994). Effect of seaweed concentrates on growth and development of the marigold Tagetes patellae. J. Appl. Physol., 6(4), 427-428.

Turner, N.C. (1981). Techniques and experimental approaches for the measurements of plant water status. Plant and soil.,58, 339-366.

Yan, J. (1993). Influence of plant growth regulators on turfgrass polar lipid composition, tolerance to drought and saline stresses, and nutrient efficiency. Ph. D. Dissertation. CSES, Virginia Tech. 
